\title{
Headache and Atypical Presentation of Motor Disorders in A Paraneoplastic Myasthenic Syndrome Secondary to Thymoma: A Case Report
}

\author{
Gabriele Cioni ${ }^{1^{*}}$ (D), Mauro Zuccotti ${ }^{1}$ (D), Fabio Daviddi ${ }^{1}$ (D), Gianluca Buffini ${ }^{1}$ (D)
}

${ }^{1}$ USL Toscana Centro, SS Cosma and Damiano Hospital, Emergency Department, Pescia (Pistoia), Italy

\section{Correspondence:}

Gabriele Cioni

Address: Emergency Department, SS Cosma and Damiano Hospital, USL Toscana Centro, 51017 Pescia (Pistoia), Italy

Email: gabrielec.83@gmail.com

Received: 29.12.2021,

Accepted: 22.01.2022

https://doi.org/10.29333/jcei/11704

\section{ABSTRACT}

Objectives: The diagnosis of early onset of myasthenia gravis in the emergency room represents a real challenge, especially when symptoms of presentation are atypical and nonspecific features.

Material and methods: We described a case of 33 years old woman who went to the Emergency Department of Pescia (Pistoia, Italy) for a spontaneous intense frontal headache associated with the presence of rhinolalia and unilateral shoulder girdle disorders, initially misdiagnosed.

Results: The atypical and initially mild presentation of the disease contributed to the delay in diagnosis; the progression of motor disturbances subsequently led to the definition of the clinical pattern. In particular, the patient showed AChR antibody positivity, associated with a thymoma.

Conclusions: Despite being a well-known pathology and widely described in the literature, myasthenia gravis at onset is still a difficult diagnosis in cases of atypical presentation or with infrequent symptoms.

Keywords: headache, paraneoplastic myasthenic syndrome, thymoma

\section{INTRODUCTION}

The diagnosis at the onset of myasthenia gravis in the emergency room represents a real challenge, especially when symptoms of presentation are atypical and non-specific features.

\section{MATERIAL AND METHODS}

In this case report, a 33 years old woman went to the Emergency Department of Pescia (Pistoia, Italy) for the onset of spontaneous intense frontal headache, which was persistent for several days, resistant to common painkillers, in the absence of a history of migraine.

Regarding her medical history, patient reported the presence of concurrent apparently non-specific disorders. In particular, she described a very mild rhinolalia started several days before, which was already evaluated by the otolaryngology specialist, and which improved after decongestant and antihistamine therapy. In addition, the patient reported asymmetrical laterocervical muscle pain for about three weeks, which was persistent despite antiinflammatory therapy. However, instrumental analysis, in particular ultrasounds, were inconclusive in identifying a clear inflammatory pattern of articular or muscular structures. Rhinolalia and laterocervical pain were not initially considered related, but secondary to sinusitis and linked to the patient's work, respectively.

\section{RESULTS}

At the emergency room visit, the headache was intense, and associated with photophobia and vomiting, despite paracetamol therapy. We applied the protocol for management of headache [1]. 
On physical examination, the patient appeared apyretic; neurological alterations, in particular meningism, visus deficit or diseases of the other cranial nerves, were excluded.

In this occasion, rhinolalia was not considered associated with headache, considering the finding of turbinate hypertrophy and congestion of the nasal mucosa following the ENT visit. Moreover, the patient described the musculoskeletal symptoms of the upper limb as mild pain, not asthenia.

Blood gas analysis was within normal limits, excluding alteration of respiratory exchanges or carbon monoxide poisoning. We ruled out alcohol and drug use. There were no signs of sinusitis at rhinoscopy. By bed-side ultrasound evaluation we excluded alterations of the afferent cerebro vessels, or of temporal artery. Subsequently, patient performed a Computed Tomography scan of the skull-brain which, in the absence of ischemic or hemorrhagic alterations, revealed an arachnoid cyst, worthy of deepening with MRI. After increasing the pain-relieving therapy, we discharged the patient with a prescription for brain MRI and subsequent re-evaluation in follow-up at two weeks.

At the check-up (15 days later), the patient showed a significantly modified clinical pattern.

In particular, she reported worsening of the disorder of the right upper limb, with progressive onset of marked asthenia, and initial involvement of the left side as well; moreover, she described difficulty walking, reporting extreme heaviness in the lower limbs. The patient was limited in both dressing and working.

The headache had subsided, but persisted despite symptomatic therapy.

Rhinolalia was always present, and indeed much more marked than in the previous finding. For the remaining neurological physical examination, we found no other significant alterations; in particular, there was no ptosis, dysphagia or alteration of skeletal reflexes. MRI, previously requested, was normal.

In consideration of the clinic pattern, we admitted the patient to the neurology department with the suspicion of myasthenia gravis. The haematological tests showed positivity for AChR antibody, confirming the diagnosis. Further investigations confirmed the presence of a mediastinal mass, which, according to further investigations, resulted in a benign thymoma. Accordingly, with current evidence [2], patients underwent surgical intervention.

\section{DISCUSSION}

Myasthenia gravis represents one of the most common autoimmune neuromuscular disorders, widely studied and well characterized, both in the etiological and pathophysiological mechanisms [3]. However, it is a relatively uncommon disorder, with a preference for the female sex [4] in the second and third decades, an annual incidence of 7 to 23 new cases per million, and a prevalence of approximately 70 to 320 per million [5,6]; for these reasons an early diagnosis at the onset could be challenging.

The main clinical features of myasthenia are extremely variable and depend both on various degrees of muscle weakness, that in the various districts involved and their association [5]. In particular, an involvement of oropharyngeal muscle may produce dysarthria and dysphagia; the speech could sound nasal or hypophonic when there is weakness of the palatal muscles [6]. An involvement of arms is more frequent than legs, with a more common weakness of proximal muscles, wrist and finger extensors. In a prospective case series was described a predominantly distal presentation [3].

In this clinical case, the onset of rhinolalia was mild, and was not associated with other muscle disorders, clearly detectable at the emergency room visit. In particular, there was no involvement of the bulbar muscles, in the absence of both ptosis and diplopia, which usually represent more than $50 \%$ of the initial presentation [4,7]. Furthermore, the finding of turbinate hypertrophy and mucosal congestion, and the partial response to antihistamine and decongestant therapy may have misled and delayed the differential diagnosis. In addition, the patient did not complain of dysarthria, dysphagia or difficulty chewing, symptoms that account for $15 \%$ at onset [8].

The symptoms of the upper right limb occurred atypically, i.e. without symmetrical onset and without involvement of the proximal limb muscles; according to current literature, limb muscle involvement represents less than $5 \%$ of cases, and presentation of the laterocervical muscles is even rarer [8]. Furthermore, due to repeated muscular efforts on the right side, it was initially attributed to the patient's occupation. A further study confirms that atypical and unusual presentations are burdened by diagnostic difficulties and delays; in particular, the isolated presentation of the limb-girdle is the most frequent atypical form [9].

Recent literature data suggest the association between myasthenia gravis and painful syndromes, such as headache, thus widening the field of action beyond the mere motor manifestations. The pathophysiological mechanism of these painful disorders should be linked to the dysfunction of the autonomic nervous system [10]. In a different study [11], the impact of headache on the quality of life of patients with myasthenia gravis was investigated. The authors described the different clinical patterns of headache, suggesting that there was no direct pathogenetic link with myasthenia gravis. Rather, they postulated that the headache was secondary to typical eye disorders, which may be persistent, but sometimes fluctuating. In the case we have described, therefore, the headache could be intercurrent with the neurological disorders found, or be secondary to a slight and fluctuating ocular disorder. Indeed, at the time of the 
emergency room visit we did not find any ocular motility disturbances in the patient, we cannot exclude intermittent disturbances. However, many other studies are still in progress.

The tests carried out on the patient showed the presence of Ach-R antibodies, which are usually associated with the presence of thymic mass. In AChR antibody-positive myasthenia gravis [12], thymic changes are extremely frequent, counting about $75 \%$ of cases, ranging from hyperplasia alone in $80 \%$ of cases, to neoplastic forms in $15 \%$ [13]. In particular, thymomas represent from 15 to $20 \%$ of mediastinal neoplasms $[2,13]$, usually clinically silent, or otherwise associated with myasthenia gravis such as paraneoplastic syndrome, equally represented in the two sexes, with a higher incidence between 40 and 60 years [13]. The atypical presentation of myasthenia gravis is usually associated with the presence of anti-MuSK antibodies, rather than anti-ACh receptor antibodies [3]. However, although infrequent, there are reports that describe this eventuality [14]. Moreover, to confirm our data, evidence from the literature reports that in subjects with positive anti-MuSK antibodies, the association with thymic pathology is negligible $[15,16]$.

The current data available in the literature do not provide univocal evidence on the association of myasthenia and thymic carcinoma or other malignant neoplasms [17-19].

The surgery intervention must be carefully evaluated, as it can lead to an improvement of the symptoms, or to their complete remission [20].

\section{CONCLUSIONS}

Despite being a well-known pathology and widely described in the literature, myasthenia gravis at onset is still a difficult diagnosis in cases of atypical presentation or with infrequent symptoms. At the end of the diagnostic course, the patient described in the clinical case was diagnosed with positive paraneoplastic Ach-R Ab myasthenic syndrome in the presence of benign thymoma with indication for surgical removal [2]. In this case, the presence of both motor disorders, much more widely described in the literature, and of painful syndromes, have contributed to the definition of the clinical pattern.

Author contributions: All authors have sufficiently contributed to the study, and agreed with the results and conclusions.

Funding: No funding source is reported for this study.

Acknowledgements: All authors would like to thank to the nurses, Noemi Timpanaro and Sabrina Ferrarassa, who assisted the authors in the clinical management of the patient at the time of access to the emergency room.

Declaration of interest: No conflict of interest is declared by authors.

\section{REFERENCES}

1. Steiner TJ, Jensen R, Katsarava Z, et al. Aids to management of headache disorders in primary care (2nd edition). J Headache Pain. 2019;20:57. doi: 10.1186/s10194-018-0899-2.

2. Comacchio GM, Marulli G, Mammana M, Natale G, Schiavon M, Rea F. Surgical decision making: Thymoma and myasthenia gravis. Thorac Surg Clin. 2019;29(2):203-13. doi: 10.1016/j.thorsurg.2018.12.007.

3. Werner P, Kiechl S, Löscher W, Poewe W, Willeit J. Distal myasthenia gravis frequency and clinical course in a large prospective series. Acta Neurol Scand. 2003;108(3):209-11. doi: 10.1034/j.16000404.2003.00136.x.

4. Grob D, Brunner N, Namba T, Pagala M. Lifetime course of myasthenia gravis. Muscle Nerve. 2008;37(2):141-9. doi: 10.1002/mus.20950.

5. Gilhus NE. Myasthenia gravis. N Engl J Med. 2016; 375:2570-81. doi: 10.1056/NEJMra1602678.

6. Carr AS, Cardwell CR, McCarron PO, McConville J. A systematic review of population based epidemiological studies in myasthenia gravis. BMC Neurol. 2010;10:46. doi: 10.1186/1471-2377-10-46.

7. Grob D, Arsura EL, Brunner NG, Namba T. The course of myasthenia gravis and therapies affecting outcome. Ann N Y Acad Sci. 1987;505:472-99. doi: 10.1111/j.17496632.1987.tb51317.x.

8. Phillips 2nd LH. The epidemiology of myasthenia gravis. Ann N Y Acad Sci. 2003;998:407-12. doi: 10.1196/annals.1254.053.

9. Rodolico C, Parisi D, Portaro S, et al. Myasthenia gravis: Unusual presentations and diagnostic pitfalls. J Neuromuscul Dis. 2016;3(3):413-8. doi: 10.3233/JND160148 .

10. Tong O, Delfiner L, Herskovitz S. Pain, headache, and other non-motor symptoms in myasthenia gravis. Curr Pain Headache Rep. 2018;22(6):39. doi: 10.1007/s11916018-0687-3.

11. Nishimoto Y, Suzuki S, Utsugisawa K, et al. Headache associated with myasthenia gravis: The impact of mild ocular symptoms. Autoimmune Dis. 2011;2011:840364. doi: 10.4061/2011/840364.

12. Castleman B. The pathology of the thymus gland in myasthenia gravis. Ann N Y Acad Sci. 1966;135(1):496505. doi: 10.1111/j.1749-6632.1966.tb45497.x.

13. Safieddine N, Liu G, Cuningham K, et al. Prognostic factors for cure, recurrence and long-term survival after surgical resection of thymoma. J Thorac Oncol. 2014;9(7):1018-22. doi: 10.1097/JTO.0000000000000215.

14. Asghar H, Sheikh FN, Dev H, Lazarevic MB, Hassan SA. An atypical presentation of myasthenia gravis: A case report. Cureus. 2019;11(4):e4563. doi: $10.7759 /$ cureus.4563. 
15. Leite MI, Ströbel P, Jones M, et al. Fewer thymic changes in MuSK antibody-positive than in MuSK antibodynegative MG. Ann Neurol. 2005;57(3):444-8. doi: 10.1002/ana.20386.

16. Díaz-Manera J, Martínez-Hernández E, Querol L, et al. Long-lasting treatment effect of rituximab in MuSK myasthenia. Neurology. 2012;78(3):189-93. doi: 10.1212/WNL.0b013e3182407982.

17. Sieb JP. Myasthenia gravis: An update for the clinician. Clin Exp Immunol. 2014;175(3):408-18. doi: 10.1111/cei.12217.
18. Levin N, Abramsky O, Lossos A, et al. Extrathymic malignancies in patients with myasthenia gravis. J Neurol Sci. 2005;237(1-2):39-43. doi: 10.1016/j.jns.2005.05.009.

19. Abrey LE. Association of myasthenia gravis with extrathymic Hodgkin's lymphoma: Complete resolution of myasthenic symptoms following antineoplastic therapy. Neurology. 1995;45(5):1019. doi: 10.1212/wnl.45.5.1019.

20. Yi JS, Guptill JT, Stathopoulos P, Nowak RJ, O'Connor $\mathrm{KC}$. B cells in the pathophysiology of myasthenia gravis. Muscle Nerve. 2018;57(2):172-84. doi: 10.1002/mus.25973. 